\title{
The order parameter and susceptibility of the 3D Ising-like system in an external field near the phase transition point
}

\author{
M.P. Kozlovskil|* R.V. Romanikł \\ Institute for Condensed Matter Physics of the National Academy of Sciences of Ukraine, \\ 1 Svientsitskii Str., 79011 Lviv, Ukraine
}

Received September 20, 2010, in final form October 1, 2010

\begin{abstract}
The present work is devoted to the investigation of the 3D Ising-like model in the presence of an external field in the vicinity of critical point. The method of collective variables is used. General expressions for the order parameter and susceptibility are calculated as functions of temperature and the external field as well as scaling functions of that are explicitly obtained. The results are compared with the ones obtained within the framework of parametric representation of the equation of state and Monte Carlo simulations. New expression for the exit point from critical regime of the order parameter fluctuations is proposed and used for the calculation.
\end{abstract}

Key words: Ising model, order parameter, collective variables, critical point

PACS: $05.50 .+q, 64.60 . F r, 75.10 . H k$

\section{Introduction}

Most problems of statistical physics are not exactly solvable. Only certain problems of physical interest have got exact analytical solutions. In particular, this is the two-dimensional Ising model [1]. However, in the presence of an external field one should resort to some approximate methods again. Thus, for theoretical description of physical phenomena two types of approaches are used. The first one is the approximate description for physically reasonable models. Such an approach does not possess a sufficient mathematical rigour. Nevertheless, it allows one to obtain qualitative, and sometimes quantitative information on the system behaviour. Another way is to carry out the investigation of either classical or quantum systems within the framework of some basic exactly solvable model systems. In many cases this is evidently an effective means. On the one hand, any mathematical model is only an approach to the real image of the physical system. On the other hand, those several models, for which rigorous mathematical estimates can be obtained, deserve careful study. An example of this way of implementation is the approximating Hamiltonian method devised by N.N. Bogolyubov (Jr.) 2-4], Subject to these models it was argued in [3], that this method gives rise to asymptotically exact solutions in the thermodynamical limit being sufficient for many applications.

Nonetheless, so far the available theoretical methods frequently produce mathematical singularities appearing in the vicinity of the second order phase transition point. Thus, the problem needs to be treated very carefully in this case. From this point of view the Yukhnovskii's method of a phase transition description [5] used in this work is quite consistent with Bogolyubov's(Jr.) ideas of using the canonical collective variable transformation approach to the corresponding Bogolyubov's functional equation [6 -8] for the correlation functions of a simple magnet system Hamiltonian instead of that for the standard Ising model. The related functional equation splitting, compatible with the Bogolyubov's principle of correlations weakening, proves to be equivalent to the suitable mean-field approximation of higher order, giving rise to a closed solution in the thermodynamical

\footnotetext{
*E-mail: mpk@icmp.lviv.ua

†E-mail: romanik@icmp.lviv.ua
} 
limit. Thereby, the both methods enable one to obtain a complete solution of the problem (at least approximately) starting with specifying a Hamiltonian and finishing with calculating thermodynamic functions [9]. In the present paper, the study of physical characteristics is performed at near phase transition temperature $T_{\mathrm{c}}$ in the presence of external field.

\section{The model}

The properties of spin systems are known to be very well described by the Ising model. The Hamiltonian of this model in the external field is written in the form

$$
H=-\frac{1}{2} \sum_{\mathbf{i}, \mathbf{j}} \Phi\left(r_{\mathbf{i}, \mathbf{j}}\right) \sigma_{\mathbf{i}} \sigma_{\mathbf{j}}-\mathcal{H} \sum_{\mathbf{i}} \sigma_{\mathbf{i}}
$$

Here $\Phi\left(r_{\mathbf{i}, \mathbf{j}}\right)$ is a short-range interaction potential between spins located at the $i$-th and $j$-th sites of a simple cubic lattice with a lattice constant $c$. The spin variables $\sigma_{\mathbf{i}}$ take on two values \pm 1 , and $\mathcal{H}$ is an external field.

The partition function of the three-dimensional (3D) Ising model can be expressed in terms of the collective variables (CV) [5]

$$
Z=\int \exp \left(\frac{1}{2} \sum_{\mathbf{k} \in \mathcal{B}} \beta \tilde{\Phi}(k) \rho_{\mathbf{k}} \rho_{-\mathbf{k}}\right) J_{h}(\rho)(\mathrm{d} \rho)^{N}
$$

where $J_{h}(\rho)$ is the transition Jacobian from the spin variables $\sigma_{\mathbf{i}}$ to the $\mathrm{CV} \rho_{\mathbf{k}}$ and $\beta=1 / k_{\mathrm{B}} T$ is the inverse temperature. The summation in (1.2) is performed over the wave vectors $\mathbf{k}$ within the first Brillouin zone

$$
\mathcal{B}=\left\{\mathbf{k}=\left(k_{x}, k_{y}, k_{z}\right) \mid k_{i}=-\frac{\pi}{c}+\frac{2 \pi}{c} \frac{n_{i}}{N_{i}} ; n_{i}=1,2, \ldots, N_{i} ; i=x, y, z\right\}
$$

that corresponds to the volume of periodicity

$$
\Lambda=\left\{\mathbf{l}=\left(l_{x}, l_{y}, l_{z}\right) \mid l_{i}=c n_{i} ; n_{i}=1,2, \ldots, N_{i} ; i=x, y, z\right\}
$$

and $N=N_{x} N_{y} N_{z}$ is the total number of spins.

In the expression for the partition function (1.2) the Fourier transform of an interaction potential arises. In the case of exponentially decreasing potential

$$
\Phi\left(r_{\mathbf{i}, \mathbf{j}}\right)=A \exp \left(-r_{\mathbf{i}, \mathbf{j}} / b\right)
$$

its Fourier transform has the form

$$
\tilde{\Phi}_{\mathrm{G}}(k)=\frac{\tilde{\Phi}(0)}{\left(1+b^{2} \mathbf{k}^{2}\right)^{2}}, \quad \tilde{\Phi}(0)=8 \pi A(b / c)^{3} .
$$

Here $b$ is the effective interaction range.

The long-wave (small values of the wave vector $\mathbf{k}$ ) fluctuations of the spin moment are known to play a crucial role in the system behaviour near the critical point. Therefore, the explicit dependence of the Fourier transform of the interaction potential is used only for small values of the wave vector. For large $\mathbf{k}, \tilde{\Phi}(k)$ is assumed to be constant. In our investigation we will use the so-called parabolic approximation for $\tilde{\Phi}_{\mathrm{G}}(k)$ with "small" values of the wave vector $\mathbf{k}$ and an averaged estimation in the short-wave region

$$
\tilde{\Phi}(k)= \begin{cases}\Phi(0)\left(1-2 b^{2} k^{2}\right), & \mathbf{k} \in \mathcal{B}_{0} \\ \Phi_{0}=\Phi(0) \bar{\Phi}, & \mathbf{k} \in \mathcal{B} \backslash \mathcal{B}_{0}\end{cases}
$$

Here the quantity $\Phi_{0}$ characterizes the part of the potential averaged with respect to large values of $\mathbf{k} \in \mathcal{B} \backslash \mathcal{B}_{0}$ which are not essential for describing the critical behaviour. However, the value $\Phi_{0}$ is essential in calculating non-universal quantities, for example, the critical temperature. 


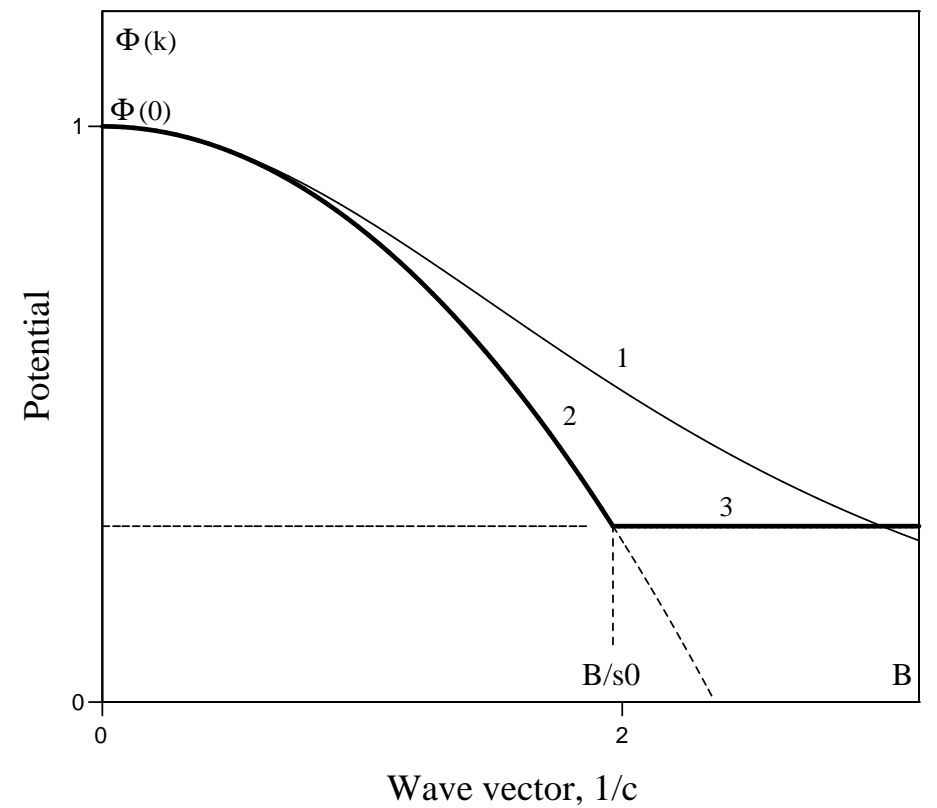

Figure 1. A sketch for the Fourier transform (1.6) of the interaction potential (1.5) (curve 1) and its parabolic approximation (1.7) (curve 2). The curve 3 corresponds to $\Phi_{0}=\Phi(0) \bar{\Phi}$ with $\bar{\Phi}$ from (1.10).

In such a way we replace (1.6) with the formula (1.7) where

$$
\mathcal{B}_{0}=\left\{\mathbf{k}=\left(k_{x}, k_{y}, k_{z}\right) \mid k_{i}=-\frac{\pi}{c_{0}}+\frac{2 \pi}{c_{0}} \frac{n_{i}}{N_{0 i}} ; n_{i}=1,2, \ldots, N_{0 i} ; i=x, y, z\right\}
$$

is the region of validity for the parabolic approximation. Equalities $N_{0 x} N_{0 y} N_{0 z}=N_{0}$ and $N_{0}=$ $N s_{0}^{-d}$ ( $d=3$ is the space dimension) take place and $s_{0} \geqslant 1$. The parameter $s_{0}$ determines an effective block lattice with a lattice constant $c_{0}=c s_{0}$. The parameter $s_{0}$ itself is determined differently for different potentials provided that in the region of $\mathbf{k} \in \mathcal{B} \backslash \mathcal{B}_{0}$ the dependence of the interaction potential on the wave vector should be the weakest. To find numerical value of $s_{0}$ we impose the condition (see figure 1)

$$
\Phi\left(B_{0}\right)=\Phi(0) \bar{\Phi} .
$$

Here $B_{0}=B / s_{0}$ with $B=\pi / c$ being the boundary of Brillouin zone. The quantity $\bar{\Phi}$ is presented in the form of two terms

$$
\bar{\Phi}=\left\langle\tilde{\Phi}_{\mathrm{G}}(k)\right\rangle_{\mathcal{B} \backslash \mathcal{B}_{0}}+\Phi_{\infty}
$$

where the first one $\left\langle\tilde{\Phi}_{\mathrm{G}}(k)\right\rangle_{\mathcal{B} \backslash \mathcal{B}_{0}}$ is the averaged value of $\Phi(k)$ in the interval $\left(B / s_{0}, B\right]$

$$
\left\langle\tilde{\Phi}_{\mathrm{G}}(k)\right\rangle_{\mathcal{B} \backslash \mathcal{B}_{0}}=\int_{\mathcal{B} \backslash \mathcal{B}_{0}} \tilde{\Phi}_{\mathrm{G}}(k) \mathrm{d} \mathbf{k} / \int_{\mathcal{B} \backslash \mathcal{B}_{0}} \Phi(0) \mathrm{d} \mathbf{k} .
$$

It results in

$$
\begin{aligned}
\left\langle\tilde{\Phi}_{\mathrm{G}}(k)\right\rangle_{\mathcal{B} \backslash \mathcal{B}_{0}}= & \frac{3}{2 \pi^{2}} \frac{s_{0}^{3}}{s_{0}^{3}-1}\left(\frac{c}{b}\right)^{3}\left[\frac{b}{c}\left(\frac{s_{0}^{-1}}{1+\left(\frac{\pi}{s_{0}}\right)^{2}\left(\frac{b}{c}\right)^{2}}-\frac{1}{1+\pi^{2}\left(\frac{b}{c}\right)^{2}}\right)\right. \\
& \left.+\frac{1}{\pi} \arctan \left(\pi \frac{b}{c}\right)-\frac{1}{\pi} \arctan \left(\frac{\pi}{s_{0}} \frac{b}{c}\right)\right] .
\end{aligned}
$$


The second term in (1.10) $\Phi_{\infty}$ is determined by the condition that $\beta_{\mathrm{c}} \Phi(0) \rightarrow 1$ when the ratio of $b / c \rightarrow \infty$. Thereby, taking into account the condition (1.9) as well as the expressions (1.7) and (1.10) we obtain the equation for finding $s_{0}$

$$
s_{0}=\pi \sqrt{2} \frac{b}{c}(1-\bar{\Phi})^{-1 / 2}
$$

As we can see, the quantity $s_{0}$ depends on the ratio of the effective range $b$ to the lattice constant $c$. The calculated values of $s_{0}$ (for different $b / c$ ) are reported in table 1 .

Table 1. Numerical values of the parameter $s_{0}$ depending on $b / c$.

\begin{tabular}{|c|c|c|c|c|c|c|c|c|c|}
\hline$b / c$ & 0.3 & 0.35 & 0.4 & 0.45 & 0.5 & 0.55 & 0.6 & 0.8 & 1.0 \\
\hline$s_{0}$ & 1.5996 & 1.7680 & 1.9429 & 2.1228 & 2.3070 & 2.4947 & 2.6853 & 3.4690 & 4.2741 \\
\hline
\end{tabular}

\section{The results of calculating the partition function in the external field}

In our calculations we will use the " $\rho^{4}$-model" approximation [5]. The functional representation for the partition function in this approximation is as follows

$$
Z=Z_{0} \int(\mathrm{d} \rho)^{N_{0}} \exp \left[a_{1} \sqrt{N_{0}} \rho_{0}-\frac{1}{2} \sum_{\mathbf{k} \in \mathcal{B}_{0}} d(k) \rho_{\mathbf{k}} \rho_{-\mathbf{k}}-\frac{a_{4}}{4 !} N_{0}^{-1} \sum_{\mathbf{k}_{i} \in \mathcal{B}_{0}} \rho_{\mathbf{k}_{1}} \ldots \rho_{\mathbf{k}_{4}} \delta_{\mathbf{k}_{1}+\ldots+\mathbf{k}_{4}}\right] .
$$

Here the quantity $d(k)$ contains the Fourier transform of the interaction potential

$$
d(k)=a_{2}+\beta \Phi(0) \bar{\Phi}-\beta \Phi(k)
$$

and expressions for $a_{n}$ have been obtained in [10].

Computation of (2.1) is performed by using the Kadanoff's idea of constructing block lattices $[11,12]$. These calculations were first realized by K.Wilson [13, 14]. In our work we will use the CV method proposed by I.Yukhnovskii [5] being generalized to the case of an external field present in [15]. Based on it, the step-by-step integration of the partition function over the layers of the CV phase space leads to the following expression

$$
Z=Z_{0}[Q(d)]^{N_{0}}\left(\prod_{n=1}^{n_{\mathrm{p}}} Q_{n}\right) Z_{\mathrm{LGR}}
$$

Here

$$
Z_{0}=2^{N}(\cosh h)^{N} \exp \left(\frac{1}{2} N \beta \Phi(0) \bar{\Phi}\right)
$$

with the dimensionless field $h=\beta \mathcal{H}$. The explicit expression for quantities $Q(d)$ and $Q_{n}$ are given in [15]. Specifically, $Q_{n}$ corresponds with the partial partition function of the $n$-th layer (or, in other words, the $n$-th block structure) and is expressed in the form

$$
Q_{n}=\left[Q\left(P_{n-1}\right) Q\left(d_{n}\right)\right]^{N_{n}}
$$

where $N_{n}=N_{0} s^{-3 n}$ with $s$ being the parameter of splitting the set of CV into subsets. Explicit expressions for $Q\left(P_{n}\right)$ and $Q\left(d_{n}\right)$ can be found in [9]. Every $Q_{n}$ is characterized by its own set of coefficients $d_{n}(k), a_{1}^{(n)}$ and $a_{4}^{(n)}$. For them, recurrence relations (RR) take place (the work [10] is devoted to this problem). By introducing notations

$$
\begin{aligned}
& d_{n}\left(B_{n+1}, B_{n}\right)=d_{n}(0)+q s^{-2 n}, \quad q=\beta \Phi(0) \bar{q} \\
& a_{1}^{(n)}=s^{-n} \omega_{n}, \quad d_{n}(0)=s^{-2 n} r_{n}, \quad a_{4}^{(n)}=s^{-4 n} u_{n}
\end{aligned}
$$


we get $\mathrm{RR}$ in the following form

$$
\begin{aligned}
\omega_{n+1} & =s^{\frac{d+2}{d}} \omega_{n}, \\
r_{n+1} & =s^{2}\left[-q+\left(r_{n}+q\right) N\left(x_{n}\right)\right], \\
u_{n+1} & =s u_{n} E\left(x_{n}\right) .
\end{aligned}
$$

Special functions $N\left(x_{n}\right)$ and $E\left(x_{n}\right)$ are presented in [15]. Their argument is

$$
x_{n}=d_{n}\left(B_{n+1}, B_{n}\right)\left(\frac{3}{a_{4}^{(n)}}\right)^{1 / 2} .
$$

One of the solutions of the RR (2.6) is the fixed point given by coordinates

$$
\omega^{*}=0, \quad r^{*}=-f_{0} \beta \Phi(0), \quad u^{*}=\phi_{0}(\beta \Phi(0))^{2} .
$$

with coefficients $f_{0}$ and $\phi_{0}[9$

$$
f_{0}=\bar{q} \frac{N\left(x^{*}\right)-1}{N\left(x^{*}\right)-s^{-2}}, \quad \phi_{0}=\frac{3(\bar{q})^{2}}{\left(x^{*}\right)^{2}}\left[\frac{1-s^{-2}}{N\left(x^{*}\right)-s^{-2}}\right]^{2}
$$

where

$$
\bar{q}=2\left(\frac{b}{c}\right)^{2} \frac{\pi^{2}}{s_{0}^{2}}\left\langle k^{2}\right\rangle_{\left(s^{-1}, 1\right]}
$$

with $s_{0}$ from (1.13).

When $n \leqslant n_{\mathrm{p}}$ the system possesses renormalization group (RG) symmetry and the solutions of $\mathrm{RR}$ can be written in the form of eigenvalue expansions

$$
\begin{aligned}
w_{n} & =s_{0}^{3 / 2} h E_{1}^{n}, \\
r_{n} & =r^{*}+c_{1} E_{2}^{n}+c_{2} R E_{3}^{n}, \\
u_{n} & =u^{*}+c_{1} R_{1} E_{2}^{n}+c_{2} E_{3}^{n}
\end{aligned}
$$

where $E_{1}$ are the eigenvalues of a matrix of RG transformation for the RR linearized near the fixed point (2.7) [16], $R=R^{(0)}\left(u^{*}\right)^{-1 / 2}$ and $R_{1}=R_{1}^{(0)}\left(u^{*}\right)^{1 / 2}$. Coefficients $c_{1}$ are defined by initial values of the quantities $\omega_{n}, r_{n}$ and $u_{n}($ at $n=0)$

$$
\omega_{0}=s_{0}^{d / 2} h, \quad r_{0}=a_{2}-\beta \Phi(0)(1-\bar{\Phi}), \quad u_{0}=a_{4} .
$$

Thus, $n_{\mathrm{p}}$ determines the number of iterations while the system belongs to the critical regime of the order parameter fluctuations. That is why the product in (2.3) is performed up to $n_{\mathrm{p}}$ only. Thereby, the quantity $n_{\mathrm{p}}$ is referred to as the exit point.

The equality $c_{1}\left(T_{\mathrm{c}}\right)=0$ coincides with the required condition for the critical temperature (in $h=0)$ to exist 9 . It yields the equation 16 .

$$
A x^{2}+B x+D=0
$$

where $x=\beta_{\mathrm{c}} \Phi(0)$ and for coefficients we have

$$
A=1-f_{0}-R^{(0)} \phi_{0}^{1 / 2}-\bar{\Phi}, \quad B=-a_{2}, \quad D=a_{4} R^{(0)} \phi_{0}^{-1 / 2} .
$$

Taking into account the above mentioned condition

$$
\lim _{b / c \rightarrow \infty} \beta_{c} \Phi(0)=1
$$

we get for $\Phi_{\infty}$

$$
\Phi_{\infty}=-f_{0}\left(1+R^{(0)} \phi_{0}^{-1 / 2} / f_{0}\right) .
$$


Further, we will consider the case when the value of quantity $x_{n}$ in the fixed point reduces to zero $\left(x^{*}=0\right)$. This is achieved by an appropriate choice of the value of the RG parameter $s=s^{*}=3.5862[9]$. Under such conditions, $E_{\mathrm{l}}$ take on numerical values as follows

$$
E_{1}=24.3549 ; \quad E_{2}=8.2551 ; \quad E_{3}=0.3763
$$

and for $R^{(0)}, R_{1}^{(0)}$ we get

$$
R^{(0)}=-0.5307, \quad R_{1}^{(0)}=0.162 .
$$

Hence, now we are able to calculate numerical values of $s_{0}$ from (1.13). The results are listed in table 1.

The last factor in (2.3) corresponds to the limit Gaussian regime (LGR) of the order parameter fluctuations in the case of $T>T_{\mathrm{c}}\left(T_{\mathrm{c}}\right.$ is the critical temperature) or to the inverse Gaussian regime (IGR) when $T<T_{\mathrm{c}}$. The explicit expression for $Z_{\mathrm{LGR}}$ is presented in [15] and for $Z_{\mathrm{IGR}}$ in [17], respectively. Especially,

$$
Z_{\mathrm{LGR}}=2^{\left(N_{n_{\mathrm{p}}+1}-1\right) / 2} Q\left(P_{n_{\mathrm{p}}}\right)^{N_{n_{\mathrm{p}}+1}} Z_{n_{\mathrm{p}}+1}
$$

where

$$
\begin{aligned}
Z_{n_{\mathrm{p}}+1}= & \int(\mathrm{d} \rho)^{N_{n_{\mathrm{p}}+1}} \exp \left(a_{1}^{\left(n_{\mathrm{p}}+1\right)} N_{n_{\mathrm{p}}+1}^{1 / 2} \rho_{0}-\frac{1}{2} \sum_{\mathbf{k} \in \mathcal{B}_{n_{\mathrm{p}}+1}} d_{n_{\mathrm{p}}+1}(k) \rho_{\mathbf{k}} \rho_{-\mathbf{k}}\right. \\
& \left.-\frac{a_{4}^{\left(n_{\mathrm{p}}+1\right)}}{4 !} N_{n_{\mathrm{p}}+1}^{-1} \sum_{\mathbf{k}_{i} \in \mathcal{B}_{n_{\mathrm{p}}+1}} \rho_{\mathbf{k}_{1}} \ldots \rho_{\mathbf{k}_{4}} \delta_{\mathbf{k}_{1}+\ldots+\mathbf{k}_{4}}\right) .
\end{aligned}
$$

In this work we make use of somewhat different (in comparison with [15-17]) expressions for the exit point

$$
n_{\mathrm{p}}=-\frac{\ln \left(\tilde{h}+h_{\mathrm{c}}\right)}{\ln E 1}-1
$$

where

$$
\tilde{h}=h s_{0}^{d / 2} / h_{0}, \quad h_{\mathrm{c}}=|\tilde{\tau}|^{p_{0}}, \quad \tilde{\tau}=\tau c_{1 k} / f_{0}
$$

with reduced temperature $\tau=\left(T-T_{\mathrm{c}}\right) / T_{\mathrm{c}}$. Quantities $h_{0}$ and $c_{1 k}$ are presented in [18, 19]. As was mentioned in [16] there is a difference between exit points in the cases of $T>T_{\mathrm{c}}$ and $T<T_{\mathrm{c}}$. This implies the presence at $T<T_{\mathrm{c}}$ of a certain internal field conjugate to the spontaneous order parameter. Let $n_{0}$ denote this difference in the absence of the external field. Therefore, in the region of temperatures less than the critical one the exit point is written

$$
n_{\mathrm{p}}^{\prime}=-\frac{\ln \left(\tilde{h}+h_{\mathrm{cm}}\right)}{\ln E 1}-1
$$

where the following notations are introduced

$$
h_{\mathrm{cm}}=\left|\tau_{1}\right|^{p_{0}}, \quad \tau_{1}=-\tau \frac{c_{1 k}}{f_{0}} E_{2}^{n_{0}}
$$

and prime denotes that $T<T_{\mathrm{c}}$ and

$$
p_{0}=\ln E_{1} / \ln E 2=\nu / \mu, \quad \mu=\frac{2}{d+2}, \quad \nu=\frac{\ln s^{*}}{\ln E_{2}}=0.605 .
$$

Here the critical exponents $\nu$ and $\mu$ describe the field (at $T=T_{\mathrm{c}}$ ) and temperature (in $h=0$ ) behaviour of correlation length, respectively. Due to (2.17) the following relationships take place

$$
\begin{array}{rlr}
s^{-\left(n_{\mathrm{p}}+1\right)}=\left(\tilde{h}+h_{\mathrm{c}}\right)^{\frac{2}{d+2}}, & E_{1}^{n_{\mathrm{p}}+1}=\left(\tilde{h}+h_{\mathrm{c}}\right)^{-1}, \\
\tilde{\tau} E_{2}^{n_{\mathrm{p}}+1}=H_{\mathrm{c}}, & H_{\mathrm{c}}=\tilde{\tau}\left(\tilde{h}+h_{\mathrm{c}}\right)^{-\frac{1}{p_{0}}}, \\
E_{3}^{n_{\mathrm{p}}+1}=H_{3}, & H_{3}=\left(\tilde{h}+h_{\mathrm{c}}\right)^{\frac{\Delta}{p_{0}}}
\end{array}
$$


where $\Delta=-\ln E_{3} / \ln E_{2}$. Similar equalities result from (2.19). In the first and the third line of (2.21) we need to substitute $n_{\mathrm{p}}$ and $h_{\mathrm{c}}$ with $n_{\mathrm{p}}^{\prime}$ and $h_{\mathrm{cm}}$, respectively. Instead of $H_{\mathrm{c}}$ we have

$$
\tilde{\tau} E_{2}^{n_{\mathrm{p}}^{\prime}+1}=-H_{\mathrm{cm}}, \quad H_{\mathrm{cm}}=-\tilde{\tau}\left(\tilde{h}+h_{\mathrm{cm}}\right)^{-\frac{1}{p_{0}}} .
$$

In contrast to recent papers [15, 17], in the present work for calculations of partition function we use slightly smaller values of the system exit point from $\mathrm{CR}$ order parameter fluctuations. In [15] the expression

$$
n_{\mathrm{p}}=-\frac{\ln \left(\tilde{h}^{2}+h_{\mathrm{c}}^{2}\right)}{2 \ln E 1}-1
$$

was used as the exit point. Here we prefer a little bit changed dependence

$$
n_{\mathrm{p}}=-\frac{\ln \left(\tilde{h}^{2}+2 \tilde{h} h_{\mathrm{c}}+h_{\mathrm{c}}^{2}\right)}{2 \ln E 1}-1
$$

that coincides with (2.17). Note that (2.23) as well as (2.24) equally well characterize the properties of the exit point from CR. Each of them in the limit of $\mathcal{H} \rightarrow 0$ takes the form [16]

$$
n_{\mathrm{p}}(\mathcal{H}=0)=m_{\tau}=-\frac{\ln |\tilde{\tau}|}{\ln E_{2}}-1
$$

and when $T=T_{\mathrm{c}}$ one obtains

$$
n_{\mathrm{p}}\left(T=T_{\mathrm{c}}\right)=n_{h}=-\frac{\ln \tilde{h}}{\ln E_{1}}-1 .
$$

Generally speaking, a choice of $n_{\mathrm{p}}$ only reshuffles the contributions in (2.3). Increasing $n_{\mathrm{p}}$ leads to an increase of the factor number in $\prod_{n=1}^{n_{\mathrm{p}}} Q_{n}$ and to a decrease of the number of variables $\rho_{\mathbf{k}}$ in the expression for $Z_{\mathrm{LGR}}$ (2.15). In the case of exact partition function calculations the total result does not depend of this. The purpose of introducing this quantity is to optimize the mathematical evaluations near phase transition point. Operating with expression (2.17) turned out to be more convenient than with (2.23) due to simplification of derivatives of free energy with respect to the field as well as to temperature. The same applies to the expression for $n_{\mathrm{p}}^{\prime}(2.19)$ that is used for a description of the model critical behaviour at $T<T_{\mathrm{c}}$.

\section{Scaling functions of the 3D Ising-like model in the vicinity of phase tran- sition point}

\subsection{Free energy}

The scheme for calculating the free energy near $T_{\mathrm{c}}$ is presented in works 15, 17] in detail. Thus, here we present only final expressions for free energy in the case of the exit points stated by (2.17) and (2.19). Let us write the free energy as a sum of three terms

$$
F=F_{\mathrm{a}}+F_{\mathrm{s}}^{( \pm)}+F_{0}^{( \pm)}
$$

where the superscript " \pm " denotes the case of $\tau>0$ and $\tau<0$, respectively. The first term is the same for the entire temperature interval and contains the analytical dependence on temperature $\tau$ and field $h$

$$
F_{\mathrm{a}}=-k_{\mathrm{B}} T N(\ln \cosh h)-\frac{1}{2} N \Phi(0) \bar{\Phi}-k_{\mathrm{B}} T N\left(\gamma_{0}+\gamma_{1} \tau+\gamma_{2} \tau^{2}\right) .
$$

All coefficients included in (3.2) are presented in 15].

The next term in (3.1) represents a non-analytical dependence of free energy on temperature and field

$$
F_{\mathrm{s}}^{( \pm)}=-k_{\mathrm{B}} T N \gamma_{\mathrm{s}}^{( \pm)}\left(\tilde{h}+h_{\mathrm{c}}\right)^{\frac{\mathrm{d} \nu}{\beta \delta}}
$$


where $\beta=\nu / 2$ is the temperature critical exponent of the order parameter and $\delta=d+2=5$ is the field one. The quantity

$$
\gamma_{\mathrm{s}}^{( \pm)}=s_{0}^{-3}\left(f_{n_{\mathrm{p}}+1}-\bar{\gamma}^{( \pm)}+f_{\mathrm{G}} / s^{3}\right)
$$

contains contributions from $\mathrm{CR}\left(\bar{\gamma}^{( \pm)}\right)$, from the so-called transition region $\left(f_{n_{\mathrm{p}}+1}\right)$ and from limit (inverse) Gaussian regime $\left(f_{\mathrm{G}}\right)$ [15 17]. One should keep in mind that in the case of $T<T_{\mathrm{c}}$ the quantity $h_{\mathrm{c}}$ is to be replaced by $h_{\mathrm{cm}}$ and the exit point $n_{\mathrm{p}}$ from (2.17) is to be replaced by $n_{\mathrm{p}}^{\prime}$ from (2.19). The quantity $\gamma_{\mathrm{s}}^{( \pm)}$depends explicitly on the scaling variable $\alpha_{0}=h / \tau^{\beta \delta}$ only, but not on $h$ or $\tau$ separately. Another scaling variable widely used in literature is $z=\tau / h^{1 / \beta \delta}$. These variables are interconnected via relationship $\alpha_{0}=z^{-\beta \delta}$.

Finally, the last contribution to the free energy (3.1) comes from the final step of integrating the partition function. It corresponds to the system free energy contribution from the collective variable $\rho_{0}$, the mean value of which is known to connect with the order parameter [5]. It has the form

$$
F_{0}^{( \pm)}=-k_{\mathrm{B}} T N\left[e_{0}^{( \pm)} h\left(\tilde{h}+h_{\mathrm{c}}\right)^{\frac{1}{\delta}}-e_{2}^{( \pm)}\left(\tilde{h}+h_{\mathrm{c}}\right)^{\frac{\mathrm{d} \nu}{\beta \delta}}\right] .
$$

Coefficients $e_{0}^{( \pm)}$and $e_{2}^{( \pm)}$are found in $[15,17]$

$$
\begin{aligned}
& e_{0}=\sigma_{0} s^{-1 / 2}, \\
& e_{2}=\frac{1}{2} \sigma_{0}^{2} s^{-3}\left(r_{n_{\mathrm{p}}+2}+\frac{1}{12} u_{n_{\mathrm{p}}+2} s_{0}^{3} \sigma_{0}^{2}\right) .
\end{aligned}
$$

where quantity $\sigma_{0}$ is a solution of a certain cubic equation

$$
\sigma_{0}^{3}+p \sigma_{0}+q=0
$$

which has been obtained from the extremum condition for $F_{0}^{( \pm)}$. Coefficients $p$ and $q$ take on the following form

$$
p=6 s_{0}^{-3} \frac{r_{n_{\mathrm{p}}+2}}{u_{n_{\mathrm{p}}+2}}, \quad q=-6 s_{0}^{-9 / 2} s^{5 / 2} \frac{h_{0}}{u_{n_{\mathrm{p}}+2}} \frac{\tilde{h}}{\tilde{h}+h_{\mathrm{c}}} .
$$

Collecting free energy contributions we arrive at

$$
F=F_{\mathrm{a}}+\Delta F_{\mathrm{s}}
$$

where $F_{\mathrm{a}}$ is given in (3.2) and singular part of free energy has the form

$$
\Delta F_{\mathrm{s}}=-k_{\mathrm{B}} T N\left[e_{0}^{( \pm)} h\left(\tilde{h}+h_{\mathrm{c}}\right)^{\frac{1}{\delta}}+\gamma_{N}\left(\tilde{h}+h_{\mathrm{c}}\right)^{\frac{\mathrm{d} \nu}{\beta \delta}}\right]
$$

and

$$
\gamma_{N}=\gamma_{\mathrm{s}}^{( \pm)}-e_{2}^{( \pm)}
$$

The term $\Delta F_{\mathrm{s}}$ plays a crucial role in describing the system behaviour near the critical point $\left(T=T_{\mathrm{c}}, h=0\right)$ and is responsible for singularities appearing therein.

Note that expression (3.10) can be presented in the form

$$
\Delta F_{\mathrm{s}}=f_{\mathrm{st}} \tau^{3 \nu}
$$

where scaling function $f_{\text {st }}$ is written as

$$
f_{\mathrm{st}}=-k_{\mathrm{B}} T N\left[\gamma_{N}(1+\alpha)^{\frac{\mathrm{d} \nu}{\beta \delta}}+\frac{h_{0}}{s_{0}^{3 / 2}} e_{0}^{( \pm)} \alpha(1+\alpha)^{\frac{1}{\delta}}\right]\left(\frac{c_{1 k}}{f_{0}}\right)^{\mathrm{d} \nu}
$$

Here we have introduced a renormalized scaling variable $\alpha$ as

$$
\alpha=\frac{\tilde{h}}{h_{\mathrm{c}}}=\frac{s_{0}^{3 / 2}}{h_{0}}\left(\frac{f_{0}}{c_{1 k}}\right)^{\beta \delta} \alpha_{0}=\frac{s_{0}^{3 / 2}}{h_{0}}\left(\frac{f_{0}}{c_{1 k}}\right)^{\beta \delta} z^{-\beta \delta} .
$$


In the absence of the external field, the second term in brackets of (3.13) disappears.

On the other hand, $\Delta F_{\mathrm{s}}$ can be expressed as

$$
\Delta F_{\mathrm{s}}=f_{\mathrm{sh}} h^{\frac{\mathrm{d} \nu}{\beta \rho}}
$$

where scaling function $f_{\text {sh }}$ is written as

$$
f_{\mathrm{sh}}=-k_{\mathrm{B}} T N\left[\gamma_{N}\left(1+\alpha^{-1}\right)^{\frac{\mathrm{d} \nu}{\beta \delta}}+e_{0}^{( \pm)}\left(1+\alpha^{-1}\right)^{\frac{1}{\delta}}\right]\left(\frac{s_{0}^{3 / 2}}{h_{0}}\right)^{\frac{\mathrm{d} \nu}{\beta \delta}}
$$

The expression (3.12) is a customary notation for the system free energy near the phase transition point in the absence of an external field. It is considered to be correct in the case of the presence of a weak field as well when, based on (3.12)-like expression, one evaluates various scaling functions. In the case of a strong field the expression (3.15) is commonly used [20]. In the case of intermediate field values $\tilde{h} \approx h_{\mathrm{c}}$, the expression (3.10) should be used.

\subsection{Order parameter}

The obtained expressions (3.1)-(3.5) enable the equation of state of the 3D Ising-like system to be established [16, 17] as well as some other system characteristics, such as susceptibility, entropy, heat capacity. This paper aims to evaluate the temperature and field dependencies of the order parameter (or, in other words, to establish the equation of state) and that of susceptibility.

In accordance with (3.1) the order parameter is presented in the form of three terms

$$
M=M_{\mathrm{a}}+M_{\mathrm{s}}^{( \pm)}+M_{0}^{( \pm)}
$$

each of which is the derivative of a certain free energy contribution with respect to the external field, so that

$$
M=-\frac{1}{N}\left(\frac{\mathrm{d} F}{\mathrm{~d} \mathcal{H}}\right)_{\mathrm{T}} .
$$

The first term $M_{\mathrm{a}}$ corresponds to the analytical part of free energy (3.2) and has the form

$$
M_{\mathrm{a}}=\tanh h \approx h .
$$

The second contribution to the order parameter $M_{\mathrm{s}}^{( \pm)}$is calculated in accordance with (3.3) and can be expressed as

$$
M_{\mathrm{s}}^{( \pm)}=\left[\frac{6}{5} \frac{s_{0}^{3 / 2}}{h_{0}} \gamma_{\mathrm{s}}^{( \pm)}+\frac{\mathrm{d} \gamma_{\mathrm{s}}^{( \pm)}}{\mathrm{d} h}\left(\tilde{h}+h_{\mathrm{c}}\right)\right]\left(\tilde{h}+h_{\mathrm{c}}\right)^{\frac{1}{\delta}}
$$

The derivative $\mathrm{d} \gamma_{\mathrm{s}}^{( \pm)} / \mathrm{d} h$ can be calculated explicitly by using formulas from 15, 17,

In order to calculate $M_{0}^{( \pm)}$we use the expression (3.5). Note that differentiating quantity $\sigma_{0}$ with respect to $h$ leads to set of terms canceling out due to (3.7). Therefore, when calculating $M_{0}^{( \pm)}$ we consider quantity $\sigma_{0}$ independent of the field. Thus, we get

$$
M_{0}^{( \pm)}=\left[e_{0}^{( \pm)}\left(1+\frac{1}{5} \frac{\tilde{h}}{\tilde{h}+h_{\mathrm{c}}}\right)-\frac{6}{5} \frac{s_{0}^{3 / 2}}{h_{0}} e_{2}^{( \pm)}-\left(\frac{d e_{2}^{( \pm)}}{\mathrm{d} h}\right)_{\sigma_{0}}\left(\tilde{h}+h_{\mathrm{c}}\right)\right]\left(\tilde{h}+h_{\mathrm{c}}\right)^{\frac{1}{\delta}} .
$$

Based on (3.20) - (3.21) we propose the following form for the equation of state

$$
M=\sigma_{00}^{( \pm)}\left(\tilde{h}+h_{\mathrm{c}}\right)^{\frac{1}{\delta}}
$$

where we have neglected $M_{\mathrm{a}}$ in comparison with other contributions to the order parameter. The scaling function $\sigma_{00}$ has the form

$$
\sigma_{00}^{( \pm)}=e_{0}^{( \pm)}\left(1+\frac{1}{5} \frac{\alpha}{1+\alpha}\right)+e_{00}^{( \pm)}+e_{02}^{( \pm)} .
$$


Here quantity $e_{0}^{( \pm)}$is defined in (3.6) and for $e_{00}^{( \pm)}, e_{02}^{( \pm)}$we find the following expressions

$$
\begin{aligned}
e_{00}^{( \pm)} & =\frac{6}{5} \frac{s_{0}^{3 / 2}}{h_{0}}\left(\gamma_{\mathrm{s}}^{( \pm)}-e_{2}^{( \pm)}\right) \\
e_{02}^{( \pm)} & =\left[\frac{\mathrm{d} \gamma_{\mathrm{s}}^{( \pm)}}{\mathrm{d} h}-\left(\frac{\mathrm{d} e_{2}^{( \pm)}}{\mathrm{d} h}\right)_{\sigma_{0}}\right]\left(\tilde{h}+h_{\mathrm{c}}\right)
\end{aligned}
$$

The first two terms on the right side of equality (3.23) depend on $\alpha_{0}$ only. For the critical amplitude $\sigma_{00}$ to be a function of only $\alpha_{0}$, it is necessary for coefficient $e_{02}^{( \pm)}$to be such a function. However, this is so because of $\gamma_{\mathrm{s}}^{( \pm)}$and $e_{2}^{( \pm)}$being functions of only $\alpha_{0}$. The derivative of such a function with respect to $h$ results in

$$
\frac{\mathrm{d} f\left(\alpha_{0}\right)}{\mathrm{d} h}=f^{\prime}\left(\alpha_{0}\right) \frac{d \alpha_{0}}{\mathrm{~d} h}=f^{\prime}\left(\alpha_{0}\right)|\tau|^{-\beta \delta}
$$

and obviously $e_{02}^{( \pm)}=e_{02}^{( \pm)}\left(\alpha_{0}\right)$.

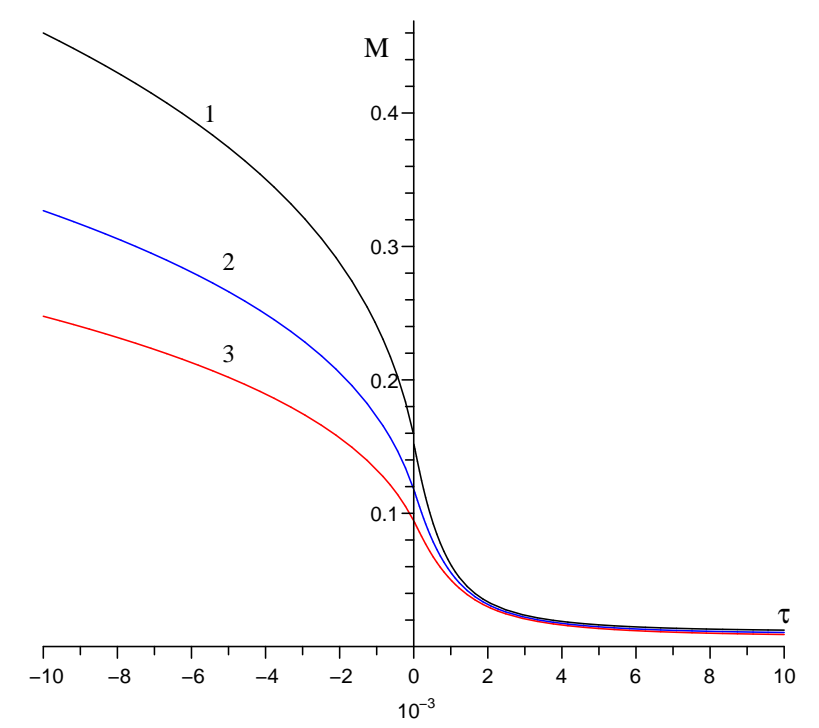

Figure 2. The order parameter as a function of $\tau$ in constant external field $h=10^{-5} .1-$ $b / c=0.3,2-b / c=0.4,3-b / c=0.5$.

The equation (3.22) permits direct crossing to be performed to the boundary cases when one of the variables (field or temperature) becomes decisive for a description of the critical behaviour. Thus, we refer to this equation as the crossover equation of state. The temperature dependence of the order parameter in the constant external field $h=10^{-5}$ is demonstrated in figure 2, that of quantity $\sigma_{00}$ from (3.23) in figure 3. Hereinafter, we use, for $h_{0}$ and $n_{0}$, the numerical values as follows [16, 17]

$$
h_{0}=0.760, \quad n_{0}=0.5
$$

Several forms of the equation of state are known to exist for Ising-like systems [21 23]. In the case of $h=0$ the order parameter exists only when $T<T_{\mathrm{c}}$ and is written as follows

$$
M=B|\tau|^{\beta}
$$




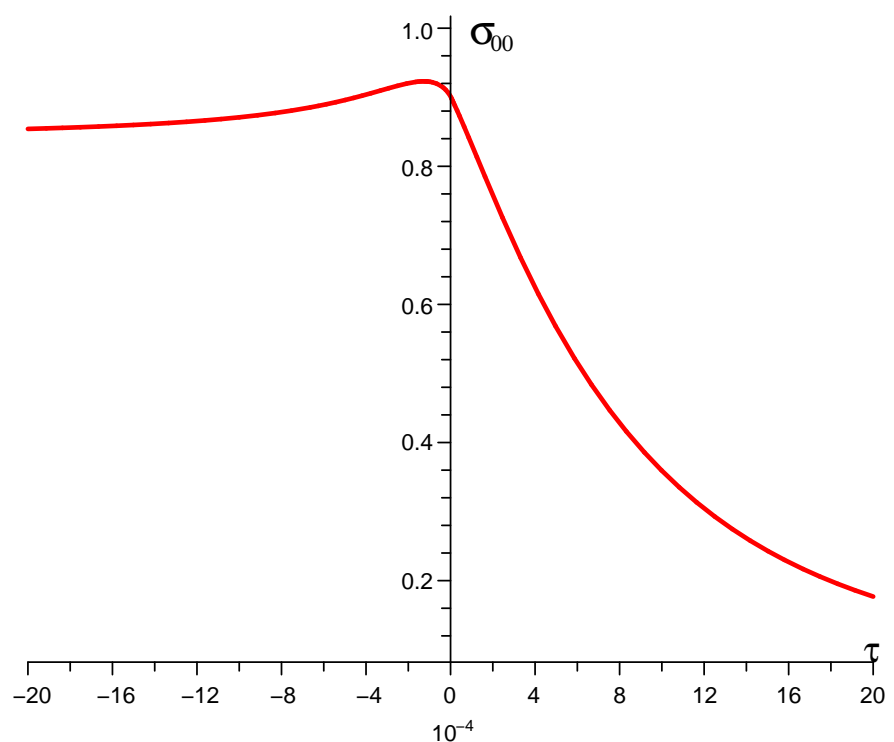

Figure 3. The scaling function $\sigma_{00}$ of the order parameter at $b / c=0.4$ as a function of the reduced temperature $\tau$ in constant external field $h=10^{-5}$.

where $B$ is the order parameter critical amplitude. In our approach, the critical amplitude $B$ takes the form

$$
B=\left.\left(c_{1 k} E_{2}^{n_{0}} / f_{0}\right)^{\beta} \sigma_{00}^{(-)}\right|_{(h=0)} .
$$

In the case of $\tau \neq 0, h \neq 0$ near the phase transition temperature $T_{\mathrm{c}}$ the equation of state is usually described by

$$
M=h^{1 / \delta} f_{\mathrm{G}}(z) .
$$

We can rewrite our equation of state (3.22) in the form of (3.28) and compare our results those of works 20, 24] where evaluation of the quantity $f_{\mathrm{G}}$ was performed. In the framework of our approach $f_{\mathrm{G}}$ is expressed as

$$
f_{\mathrm{G}}=\sigma_{00}^{( \pm)}\left(1+\alpha^{-1}\right)^{1 / \delta}\left(s_{0}^{3 / 2} / h_{0}\right)^{1 / \delta} .
$$

In figure 4 the scaling function $f_{\mathrm{G}}$ is shown as a function of $z$ for different values of the ratio $b / c$. The thick line means the parametric representation of the equation of state from [24]

$$
\begin{aligned}
M & =m_{0} R^{\beta} \Theta, \\
\tau & =R\left(1-\Theta^{2}\right), \\
h & =H_{0} R^{\beta \delta} \hat{h}(\Theta)
\end{aligned}
$$

normalized by conditions

$$
M(\tau=0)=h^{1 / \delta}, \quad \text { and } \quad M(h=0)=(-\tau)^{\beta} .
$$

The function $\hat{h}(\Theta)$ was approximated in [24] by the following polynomial

$$
\hat{h}(\Theta)=\Theta-0.76201 \Theta^{3}+0.00804 \Theta^{5} .
$$

The zeros of this function correspond to $h=0$ and $\tau>0$ (the first one $\Theta=0$ ) or $\tau<0$ (the second one $\left.\Theta_{0}=1.154\right)$. For $\Theta=1$ one obtains $\tau=0$. Therefore, normalization constants $H_{0}, m_{0}$ take on the form

$$
m_{0}=\frac{\left(\Theta_{0}^{2}-1\right)^{\beta}}{\Theta_{0}}, \quad H_{0}=\frac{m_{0}^{\delta}}{\hat{h}(1)} .
$$




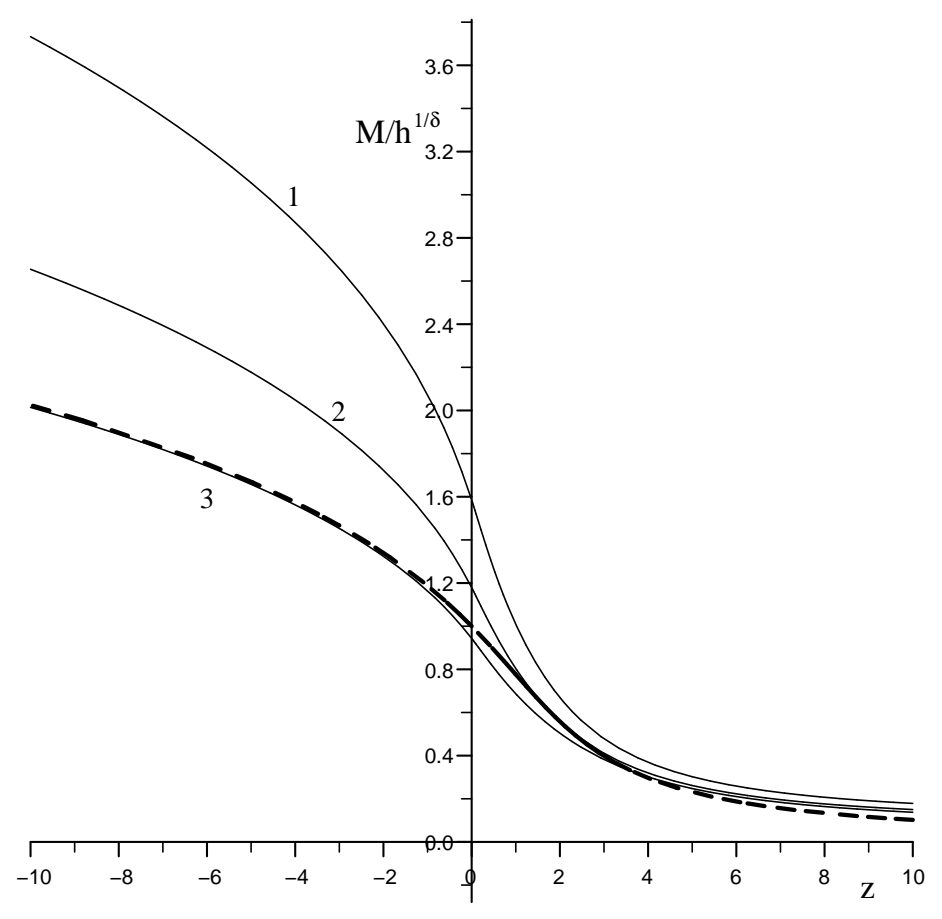

Figure 4. Scaling function $f_{\mathrm{G}}$ of the order parameter as a function of $z$ for different values of $b / c .1-b / c=0.3,2-b / c=0.4,3-b / c=0.5$. Thick dashed line shows the parametrization from (3.30) 20, 24].

The scaling function $f_{\mathrm{G}}$ and its argument $z$ are then represented in terms of new variable $\Theta$ as

$$
z=\frac{1-\Theta^{2}}{\Theta_{0}^{2}-1} \Theta_{0}^{1 / \beta}\left(\frac{\hat{h}(\Theta)}{\hat{h}(1)}\right)^{-1 / \beta \delta}, \quad f_{\mathrm{G}}=\Theta\left(\frac{\hat{h}(\Theta)}{\hat{h}(1)}\right)^{-1 / \delta} .
$$

In our investigation we do not impose any normalized conditions for the scaling functions. However, for $b / c=0.5$ we observe a good agreement of our results for $f_{\mathrm{G}}(z)$ with parametric representation results (see figure 4 and $B=1.064$ ). In [20] an excellent agreement between the Monte Carlo simulations data and the parametrization from (3.34) has been obtained.

\subsection{Susceptibility}

The system susceptibility is found by means of direct differentiation of the equation of state with respect to the external field $h$. We have

$$
\chi=\beta\left(\tilde{h}+h_{\mathrm{c}}\right)^{\frac{1}{\delta}}\left(\frac{\mathrm{d} \sigma_{00}^{( \pm)}}{\mathrm{d} h}+\frac{1}{d+2} \sigma_{00}^{( \pm)} \frac{s_{0}^{3 / 2}}{h_{0}}\left(\tilde{h}+h_{\mathrm{c}}\right)^{-1}\right) .
$$

Based on the expression for $\sigma_{00}^{( \pm)}(3.23)$ we can calculate its derivative with respect to the field $h$. Let us do it for each term separately. We get

$$
\begin{aligned}
& \left(\frac{\mathrm{d} \sigma_{00}^{( \pm)}}{\mathrm{d} h}\right)_{1}=\left(1+\frac{1}{5} \frac{\tilde{h}}{\tilde{h}+h_{\mathrm{c}}}\right) \frac{\mathrm{d} e_{0}^{( \pm)}}{\mathrm{d} h}+\frac{1}{5} \frac{s_{0}^{3 / 2}}{h_{0}} e_{0}^{( \pm)} \frac{h_{\mathrm{c}}}{\left(\tilde{h}+h_{\mathrm{c}}\right)^{2}} \\
& \left(\frac{\mathrm{d} \sigma_{00}^{( \pm)}}{\mathrm{d} h}\right)_{2}=\frac{6}{5} \frac{s_{0}^{3 / 2}}{h_{0}}\left(\frac{\mathrm{d} \gamma_{\mathrm{s}}^{( \pm)}}{\mathrm{d} h}-\frac{\mathrm{d} e_{2}^{( \pm)}}{\mathrm{d} h}\right)
\end{aligned}
$$


and

$$
\left(\frac{\sigma_{00}^{( \pm)}}{\mathrm{d} h}\right)_{3}=\frac{\mathrm{d}}{\mathrm{d} h}\left(\frac{\mathrm{d} \gamma_{\mathrm{s}}^{( \pm)}}{\mathrm{d} h}-\left(\frac{\mathrm{d} e_{2}^{( \pm)}}{\mathrm{d} h}\right)_{\sigma_{0}}\right)+\frac{s_{0}^{3 / 2}}{h_{0}}\left(\frac{\mathrm{d} \gamma_{\mathrm{s}}^{( \pm)}}{\mathrm{d} h}-\left(\frac{\mathrm{d} e_{2}^{( \pm)}}{\mathrm{d} h}\right)_{\sigma_{0}}\right) .
$$

It is important to emphasize that in (3.36) 3.37) one should take into account the dependence of $\sigma_{0}$ on the field while differentiating by $h$. But in (3.38) such a dependence is considerable only for the second derivative.

For convenience, the full expression for the derivative is written as follows:

$$
\frac{\mathrm{d} \sigma_{00}^{( \pm)}}{\mathrm{d} h}=\chi_{01}^{( \pm)}\left(\tilde{h}+h_{\mathrm{c}}\right)^{-1}
$$

where

$$
\chi_{01}^{( \pm)}=\chi_{011}^{( \pm)}+\chi_{012}^{( \pm)}+\chi_{013}^{( \pm)}
$$

and for the terms in the last equality the following expressions are valid

$$
\begin{gathered}
\chi_{011}^{( \pm)}=\frac{1}{5} e_{0}^{( \pm)} \frac{s_{0}^{3 / 2}}{h_{0}} \frac{1}{1+\alpha}, \\
\chi_{012}^{( \pm)}=\left[\left(1+\frac{1}{5} \frac{\alpha}{1+\alpha}\right) \frac{\mathrm{d} e_{0}^{( \pm)}}{\mathrm{d} h}+\frac{s_{0}^{3 / 2}}{h_{0}}\left(\frac{11}{5} \frac{\mathrm{d} \gamma_{\mathrm{s}}^{( \pm)}}{\mathrm{d} h}-\frac{6}{5} \frac{\mathrm{d} e_{2}^{( \pm)}}{\mathrm{d} h}-\left(\frac{\mathrm{d} e_{2}^{( \pm)}}{\mathrm{d} h}\right)_{\sigma_{0}}\right)\right]\left(\tilde{h}+h_{\mathrm{c}}\right)^{-1},
\end{gathered}
$$

and

$$
\chi_{013}^{( \pm)}=\left(\tilde{h}+h_{\mathrm{c}}\right)^{2} \frac{\mathrm{d}}{\mathrm{d} h}\left[\frac{\mathrm{d} \gamma_{\mathrm{s}}^{( \pm)}}{\mathrm{d} h}-\left(\frac{\mathrm{d} e_{2}^{( \pm)}}{\mathrm{d} h}\right)_{\sigma_{0}}\right] .
$$

For susceptibility we have eventually obtained

$$
\frac{\chi}{\beta}=\chi_{00}^{( \pm)}\left(\tilde{h}+h_{\mathrm{c}}\right)^{\frac{1}{\delta}-1}
$$

where the scaling function for susceptibility has the form

$$
\chi_{00}^{( \pm)}=\chi_{01}^{( \pm)}+\frac{1}{5} \frac{s_{0}^{3 / 2}}{h_{0}} \sigma_{00}^{( \pm)}
$$

In figure 5 the susceptibility is graphically presented as a function of temperature at $h=10^{-5}$.

The expression (3.44) differs from that used in [20, 22]. In the vicinity of the critical point, susceptibility is considered to have the following form

$$
\frac{\chi}{\beta}=h^{1 / \delta-1} f_{\chi}(z)
$$

where the quantity $f_{\chi}(z)$ is the susceptibility scaling function. The simple relation is known to exist between the susceptibility scaling function $f_{\chi}$ and the order parameter scaling function $f_{\mathrm{G}}$

$$
f_{\chi}(z)=\frac{1}{\delta}\left(f_{\mathrm{G}}(z)-\frac{z}{\beta} f_{\mathrm{G}}^{\prime}(z)\right)
$$

Adapted to our approach, $f_{\chi}$ takes the form

$$
f_{\chi}=\chi_{00}^{( \pm)}\left(1+\alpha^{-1}\right)^{1 / \delta-1}\left(\frac{s_{0}^{3 / 2}}{h_{0}}\right)^{1 / \delta-1} .
$$

In figure 6 this quantity is presented as a function of the scaling variable $z$. The thick line corresponds to the parametrization of [24]. In [20] one can also find Monte Carlo simulations data for $f_{\chi}(z)$ according to the parametrization of [24]. 


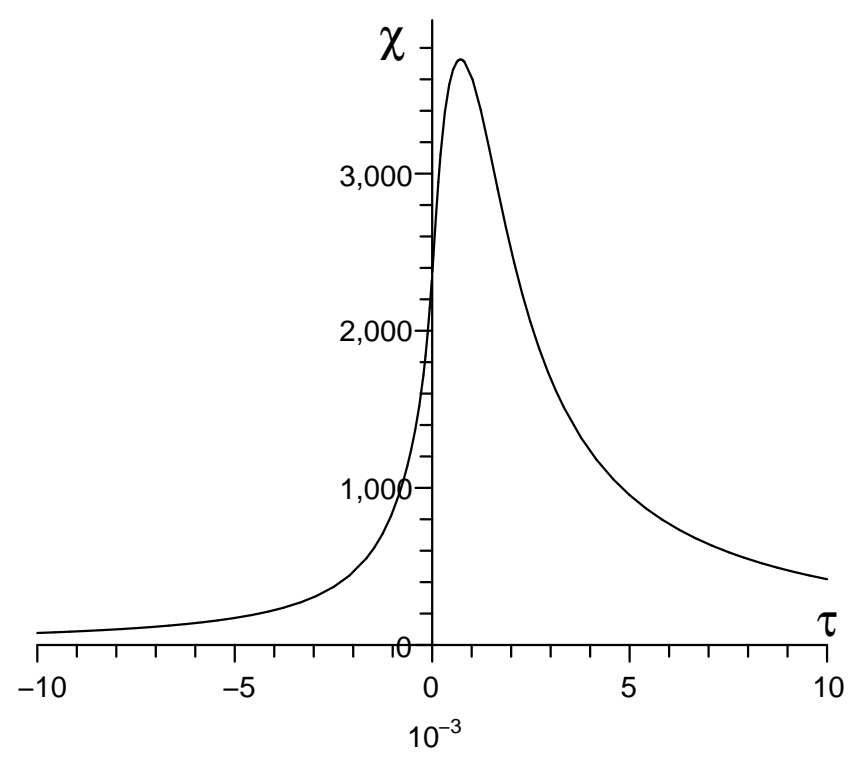

Figure 5. Susceptibility as a function of reduced temperature $\tau=\left(T-T_{\mathrm{c}}\right) / T_{\mathrm{c}}$ in constant external field $h=10^{-5}$ at $b / c=0.4$.

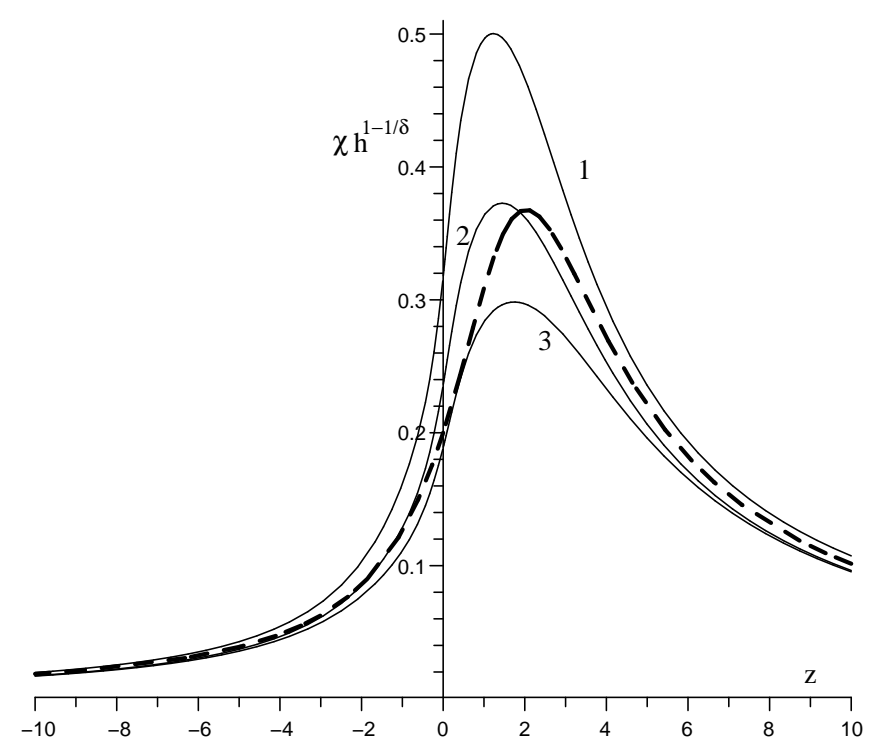

Figure 6. Scaling function of susceptibility $f_{\chi}$ as a function of the scaling variable $z=\tau / h^{1 / \beta \delta}$ : at $b / c=0.3$ (curve 1), at $b / c=0.4$ (curve 2) and at $b / c=0.5$ (curve 3). Thick dashed line shows the parametric representation from 24$]$.

\section{Conclusions}

In the present work we investigate the 3D Ising-like model on a simple cubic lattice. The research is carried out using the formalism of collective variables and in the framework of " $\rho^{4}$-model" approximation. The critical exponent $\eta$ is considered to be equal to zero. Our approach also neglects the corrections to scaling but the main critical exponents take on non-classical values. However, we have obtained explicit expressions for physics quantities as functions of reduced temperature 
$\tau$, external field $h$ and microscopic parameters of the model (the ratio of the effective range of interaction $b$ to the lattice constant $c$ ). Namely, we have calculated the free energy as well as the order parameter and susceptibility. Some results are demonstrated graphically as well.

\title{
References
}

1. Onsager L., Phys. Rev., 1944, 65, 117.

2. Bogolyubov N.N. (Jr.), A method of model Hamiltonians investigation, Nauka, 1974 (in Russian).

3. Bogolyubov N.N. (Jr.) et al., Uspekhi Mat. Nauk, 1984, 39, 3 (in Russian).

4. Seke J., Soldatov A.V., Bogolyubov N.N.(Jr.), Phys. Lett. A, 1997, 236, 261.

5. Yukhnovskii I.R., Phase Transitions of the Second Order. Collective Variables Method. World Scientific, Singapore, 1987.

6. Bogolyubov (Jr.) N.N., Prykarpatsky A.K., Phys. Part. Nuclei, 1986, 17, 789.

7. Bogolyubov (Jr.) N.N., Prykarpatsky A.K., Nonlinear Oscil., 2007, 10, 32.

8. Bogolyubov (Jr.) N.N., Prykarpatsky A.K., Theor. Math. Phys., 1986, 66, 463.

9. Yuchnovskii I.R., Kozlovskii M.P., Pylyuk I.V., Microscopic Theory of Phase Transition in the three dimensional systems. Eurosvit, Lviv 2001, (in Ukrainian).

10. Kozlovskii M.P., Condens. Matter Phys., 2005, 8, 473.

11. Kadanoff L.P., Physics (N.Y.), 1966, 2, 263.

12. Kadanoff L.P., Rev. Mod. Phys., 1967, 39, 395.

13. Wilson K.G., Phys. Rev. B, 1971, 4, 3174.

14. Wilson K.G., Kogut J., Phys. Rep., 1974, C 12, 75.

15. Kozlovskii M.P., Condens. Matter Phys., 2009, 12, 151.

16. Kozlovskii M.P., Ukr. Fiz. Zh./Reviews (Ukr. ed.), 2009, 5, 61, (in Ukrainian).

17. Kozlovskii M.P., Romanik R.V., J. Phys. Stud., 2009, 13, 4007.

18. Yukhnovskii I.R, Kozlovskii M.P., Pylyuk I.V., Phys. Rev. B, 2002, 66, 134410.

19. Kozlovskii M.P., Pylyuk I.V., Prytula O.O., Phys. Rev. B, 2006,73, 174406.

20. Engels J., Fromme J., Seniuch M., Nucl. Phys. B, 2003, 655, 277.

21. Widom B.J., J. Chem. Phys., 1965, 43, 3898.

22. Guida R., Zinn-Justin J., Nucl. Phys. B, 1977, 489, 626.

23. Hasenbusch M.J., Phys. A, 1999, 32, 4/851.

24. Zinn-Justin J., Phys. Rept., 2001, 344, 195.

\section{Розрахунок параметра порядку та сприйнятливості тривимірної Ізингоподібної моделі поблизу точки фазового переходу методом колективних змінних}

\author{
М.П. Козловський, Р.В. Романік \\ Інститут фізики конденсованих систем НАН України, 79011 Львів, вул. Свєнціцького, 1
}

Робота присвячена дослідженню тривимірної Ізингоподібної моделі поблизу критичної точки при наявності зовнішнього поля. Використовується метод колективних змінних. Розраховано загальні вирази для параметра порядку і сприйнятливості системи як функції температури і зовнішнього поля, а також скейлінгові функції цих величини отримані в явному вигляді. Зроблено порівняння з результатами, що були отримані в рамках параметричного представлення рівняння стану і методами Монте Карло симуляцій. Запропонований і використаний при обчисленнях новий вираз для точки виходу з критичного режиму флуктуацій параметра порядку.

Ключові слова: модель Ізинга, параметр порядку, колективні змінні, критична точка 
